\title{
Valine-based biphenylsulphonamide matrix metalloproteinase inhibitors as tumor imaging agents ${ }^{*}$
}

\author{
Ruth Oltenfreiter $^{\mathrm{a}}$, Ludovicus Staelens ${ }^{\mathrm{a}}$, Veerle Kersemans ${ }^{\mathrm{a}}$, Bart Cornelissen ${ }^{\mathrm{a}}$, Francis Frankenne ${ }^{\mathrm{b}}$, Jean-Michel \\ Foidart $^{\mathrm{b}}$, Christophe Van de Wiele ${ }^{\mathrm{c}}$, Guido Slegers ${ }^{\mathrm{a}}$ \\ ${ }^{a}$ Faculty of Pharmaceutical Sciences, Department of Radiopharmacy, Ghent University, Harelbekestraat 72, 9000 Ghent, Belgium \\ ${ }^{b}$ Laboratory of Tumor and Developmental Biology, University of Liège, Sart-Tilman, Liège, Belgium
}

${ }^{\mathrm{c}}$ Division of Nuclear Medicine, Gent University Hospital, De Pintelaan 185, 9000 Gent, Belgium

\begin{abstract}
Among matrix metalloproteinases (MMPs), the subfamily of gelatinases (MMP-2, MMP-9) is of particular interest due to their ability to degrade type IV collagen and other non-fibrillar collagen domains and proteins such as fibronectin and laminin. Whilst malignant cells often over-express various MMPs, the gelatinases have been most consistently detected in malignant tissues and associated with tumor growth, metastatic potential and angiogenesis. Radiosynthesis of carboxylic (1') and hydroxamic (2') MMPIs resulted in radiochemical yields of $70 \pm 5 \%(n=6)$ and $60 \pm 5 \%(n=4)$, respectively. Evaluation in A549-inoculated athymic mice showed a tumor uptake of $2.0 \pm 0.7 \% \mathrm{ID} / \mathrm{g}(3 \mathrm{~h}$ p.i.), a tumor/blood ratio of 0.5 and a tumor/muscle ratio of 4.6 at $48 \mathrm{hp}$.i. for $1^{\prime}$. For compound $2^{\prime}$ a tumor uptake of $0.7+0.2 \% \mathrm{ID} / \mathrm{g}$ ( $3 \mathrm{~h}$ p.i.), a tumor/blood ratio of 1.2 and a tumor/muscle ratio of 1.8 at $24 \mathrm{~h}$ p.i. were observed. HPLC analysis of the blood (plasma) showed no dehalogenation or other metabolites of 1' 2 h p.i. For compound 2', 65.4\% of intact compound was found in the blood (plasma) and one polar metabolite (31\%) was detected whereas in the tumor $91.8 \%$ of the accumulated activity was caused by intact compound and only $8.1 \%$ by the metabolite. Planar imaging, using a Toshiba GCA-9300A/hg SPECT camera, showed that tumor tissue could be visualized and that image quality improved by decreasing specific activity resulting in lower liver uptake, indicating some degree of saturable binding in the liver. In vivo evaluation of these radioiodinated carboxylic and hydroxamic MMP inhibitor tracers revealed that MMP inhibitors could have potential as tumor imaging agents, but that further research is necessary.
\end{abstract}

Keywords: Radiolabeled MMP inhibitors; Iodine-123; In vivo biodistribution; Tumor imaging

\section{Introduction}

Matrix metalloproteinases (MMPs) are a family of endopeptidases comprising over 20 enzyme subtypes that degrade extracellular matrix (Edwards and Murphy, 1998). Their activities are tightly controlled at the levels of gene transcription, zymogen activation by proteolysis and inhibition of active forms by the tissue inhibitors of matrix metalloproteinases (TIMPs) (Edwards et al., 1996). Among MMPs, the subfamily of gelatinases (MMP2, MMP-9) is of particular interest due to their ability to degrade type IV collagen and other non-fibrillar collagen domains and also proteins such as fibronectin and laminin. Whilst malignant cells often over-express various MMPs, the gelatinases have been most consistently detected in malignant tissues and associated with tumor growth, metastatic potential and angiogenesis (Edwards et al., 1996; Bernhard et al., 1994; Davies et al., 1993; Heppner et al., 1996; Kossakowska et al., 1992; MacDougall and Matrisian, 1995; Bremer et al., 2001; Fang et al., 2000; Murray et al., 1996; Folkman, 1997; Rudek et al, 2002).

Examples of peptidic and non-peptidic MMP inhibitors have proliferated in the literature over the last several years, with the vast majority of these compounds bearing a hydroxamic acid moiety as the requisite zinc-binding ligand. Other zinc-binding ligands that have been utilized include reverse hydroxamic acids, carboxylic acids, thiols, and thiadiazoles (Skiles et al., 2001). Tryptophane-based biphenylsulphonamide matrix metalloproteinase inhibitors were synthesized, evaluated in vitro; in vivo and as imaging agent and were published previously (Oltenfreiter et al., 2004, 2005a). Structural changes made to the biphenyl ring system, the $\alpha$-position to carboxylic acid and the carboxylate moiety, have an influence on biological activity (O'Brien et al., 2000). Earlier experiments and SAR studies showed that biphenylsulfonamides substituted with bromine in the 4'-position significantly improved the in vitro activity and exhibited superior pharmacokinetics $\left(C_{\max }, t_{1 / 2}\right.$, AUCs) relative to the compounds without bromine (Skiles et al., 2001). Halogens appended to the 4'-position of the

\footnotetext{
* Supported by EU Grant LSHC-CT-2003-503297 'cancerdegradome'.
} 
biphenyl ring, in general, increased potency against some MMPs. The magnitude of the increase did not change as the size of the halo substituent was increased from $\mathrm{F}$ to $\mathrm{Cl}$ to $\mathrm{Br}$. On the other hand varying the lipophilicity of the $\alpha$-position in general, maintained potency but decreased the oral bioavailability and significantly influenced the pharmacokinetic activity of the compounds. Replacement of for example an isobutyl group with smaller, less lipophilic substituents (methyl, hydrogen) or with a larger, more lipophilic group (benzyl) diminished the $C_{\max }$, $t_{1 / 2}$, and AUCs relative to the analogues with the isobutyl group. Incorporation of side chains bearing larger substituents gave compounds that, in general, were poorly absorbed (O'Brien et al., 2000). It has also been shown that although carboxylates exhibit weaker zinc binding properties than hydroxamic acids, they are known to show a better oral bioavailability and are less prone to metabolic degradation (Kontogiorgis et al., 2005).

In addition to potency and bioavailability, the issue of the desired selectivity profile for MMP inhibition remains an unresolved problem. Since MMP activity is an integral part of normal tissue remodelling, the possibility exists that the inhibition of particular MMPs would be detrimental and dose-limiting in a clinical setting. Doserelated side effects include arthralgia and body aches. The origin of these musculoskeletal side effects is at present undetermined, although several hypotheses have been put forth. Both the inhibition of MMP-1 and the inhibition of tumor necrosis factor (TNF) receptor shedding have been proposed as mechanisms for the origin of this troublesome complication (Skiles et al., 2001). Taking these reflections into account the Valine analogues of the earlier published Tryptophane-based biphenylsulphonamide matrix metalloproteinase inhibitors have been synthesized and evaluated. By synthesis of the analogues we aimed at producing different selectivity profiles, biological activity and pharmacokinetic properties.

This study reports on the biodistribution of iodine-123 labeled biphenylsulphonamide analogues, developed from structure-activity data of compounds with low nanomolar $\mathrm{IC}_{50}$ potencies for gelatinases (Tamura et al., 1998; Hanessian et al., 2001). These compounds were synthesized via a modified method obtained from the literature (Oltenfreiter et al., 2004; O'Brien et al., 2000; Tamura et al., 1998; Hanessian et al., 2001; Oltenfreiter et al., 2005b), and evaluated in A549-inoculated mice. Metabolic studies were performed and their suitability as diagnostic agents evaluated.

\section{Materials and methods}

\subsection{General}

Synthesis, radiosynthesis and determination of biological activity of biphenylsulphonamide derivatives (Fig. 1) was performed as reported previously (Oltenfreiter et al., 2004; O'Brien et al., 2000; Hanessian et al., 2001; Oltenfreiter et al., 2005b) 4'-iodo-biphenyl was purchased from Daniels Fine Chemicals Ltd. (Edmonton, Alb., Canada) and used for synthesis of the non-radioactive analogues.

\subsection{Radio synthesis}

The tracers were purified using an Alltech Alltima C18 column $(250 \times 4.6 \mathrm{~mm}, 5 \mu \mathrm{m})$ and a Gilson 307 pump. The eluate was monitored with an UV-VIS detector at $\lambda=254 \mathrm{~nm}$ (2487 dual $\lambda$ absorbance detector, Waters) and a NaI detector (Bicron FrisktechTM, probe 1 X 1 inch). The obtained radioactive solution was dissolved in ethanol/water $(200 \mu \mathrm{L}, 5 / 95)$ and $740 \mathrm{kBq}(20 \mu \mathrm{Ci})$ of tracer was injected for in vivo biodistribution experiments.

Fig. 1: Structure of the synthesized organic compounds.

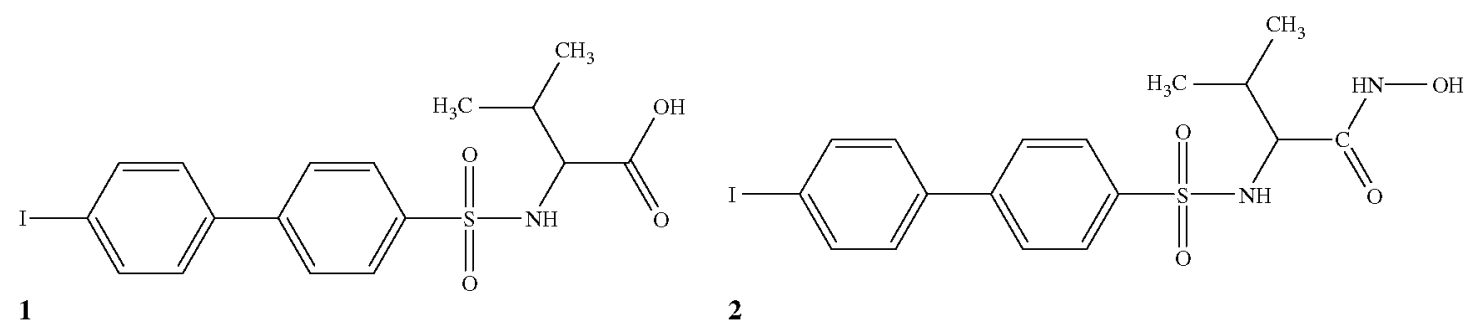

$\mathrm{R}=\mathrm{I}=1 / 2$

$\mathrm{R}={ }^{123} \mathrm{I}=1^{\prime} / 2^{\prime}$ 


\subsubsection{2-(4'-[ ${ }^{123}$ I]iodo-biphenyl-4-sulphonylamino )-3-methyl-butyric acid (1')}

To a solution of the tributylstannyl precursor $(622 \mu \mathrm{g}, 1 \mu \mathrm{mol})$ in ethanol $(130 \mu \mathrm{L})$, n.c.a. $\left[{ }^{123} \mathrm{I}\right] \mathrm{NaI}$ in sodium hydroxide solution $(15 \mu \mathrm{L} 0.05 \mathrm{M})$, chloramine $\mathrm{T}(1 \mu \mathrm{L}, 0.1 \mathrm{M}$ solution) and glacial acetic acid $(5 \mu \mathrm{L})$ were added respectively and the resulting mixture was stirred for $5 \mathrm{~min}$ at RT. The reaction mixture was quenched by addition of sodium metabisulfite $(1 \mu \mathrm{L}, 0.2 \mathrm{M}$ solution). The mixture was purified by HPLC with ethanol/phosphate buffer $(0.05 \mathrm{M}$, pH 2) (49/51) as mobile phase at a flow rate of $1 \mathrm{~mL} / \mathrm{min}$. The radiolabeled product was collected $\left(R_{t} 29.97 \mathrm{~min}\right)$ and analysed with the same HPLC system. The radiochemical yield was 70 $\pm 5 \%(n=6)$, the radiochemical purity $>98 \%$ and the specific activity $>50 \mathrm{Ci} / \mu \mathrm{mol}$.

\subsubsection{2-(4'-[ $\left.{ }^{123} I\right]$ iodo-biphenyl-4-sulphonylamino)-3-methyl-butyramide (2')}

To a solution of 2-(4' -tributylstannyl-biphenyl-4-sulphony-lamino)-3-methyl-N-trityloxy-butyramide (400 $\mu \mathrm{g}$, $0.45 \mu \mathrm{mol})$ in ethanol $(130 \mu \mathrm{L})$, n.c.a. $\left[{ }^{123} \mathrm{I}\right] \mathrm{NaI}$ in sodium hydroxide solution $(15 \mu \mathrm{L}, 0.05 \mathrm{M})$, chloramine $\mathrm{T}(1$ $\mu \mathrm{L}, 0.1 \mathrm{M}$ solution) and glacial acetic acid $(5 \mu \mathrm{L})$ were added, respectively, and the resulting mixture was stirred for $5 \mathrm{~min}$ at RT. The reaction mixture was quenched by addition of sodium metabisulfite ( $1 \mu \mathrm{L}, 0.2 \mathrm{M}$ solution). The resulting mixture of radiolabeled product was analyzed by HPLC with ethanol/ phosphate buffer $(0.05 \mathrm{M}$, pH 6) (70/30) as mobile phase at a flow rate of $1 \mathrm{~mL} / \mathrm{min}\left(R_{t} 22.5 \mathrm{~min}\right)$. In a one-pot reaction, trifluoroacetic acid $(30 \mu \mathrm{L})$ was added to $2-\left(4^{\prime}-{ }^{I 23} I\right]$ iodo-biphenyl-4-sulphonylamino)-3-methyl-N-trityloxy-butyramide and stirred for $1 \mathrm{~h}$ to obtain compound $2^{\prime}$. This second mixture was purified under the same HPLC conditions using an ethanol/phosphate buffer $(0.05 \mathrm{M}, \mathrm{pH} 6)(46 / 54)$ as mobile phase. The radiolabeled product $2^{\prime}$ was collected $\left(R_{t}\right.$ $20.69 \mathrm{~min})$ and analyzed with the same HPLC system. The radiochemical yield was $60 \pm 5 \%(n=4)$, the radiochemical purity $>98 \%$ and the specific activity $>50 \mathrm{Ci} / \mu \mathrm{mol}$.

\subsubsection{Human serum albumin}

Radioiodination of human serum albumin (HSA) with ${ }^{123}$ I (Nordion Europe, Belgium) was performed by electrophilic substitution using the Iodogen technique. Briefly: in the Iodogen vial, ${ }^{123} \mathrm{I}(111 \mathrm{MBq} ; 3 \mathrm{mCi}) \mathrm{was}$ added to HSA $(50 \mu \mathrm{g})$ in $140 \mu \mathrm{l} 0.1 \mathrm{M}$ potassium phosphate buffer at $\mathrm{pH} 8.5$. After 15 min at room temperature, the mixture was removed from the Iodogen coated vial to stop the reaction. To remove the free ${ }^{123} \mathrm{I}$ from $\left[{ }^{123} \mathrm{I}\right]$ iodo-HSA, the mixture was applied to a $0.22 \mu \mathrm{m} \mathrm{Ag-filter.} \mathrm{The} \mathrm{specific} \mathrm{activity} \mathrm{was} 1.5 \times 10^{5} \mathrm{GBq} / \mathrm{mmol}$ (4Ci/ $\mu \mathrm{mol})$. Quality control was achieved using a standard prepacked PD-10 SEC column (Size Exclusion Chromatography, Amersham Pharmacia Biotech, Sweden) using PBS/HSA as the eluting agent.

\subsection{In vitro enzyme assays of sulphonamide analogues}

\subsubsection{Enzymes}

The recombinant catalytic domain of MT1-MMP(cMTl), MT3-MMP(cMT3) and pro-MMP-2 were produced in Liège (Lang et al., 2004). Pro-MMP-9 was purchased from R\&D Systems. Activation of pro-MMP-2 and proMMP-9 was achieved by incubating pro-MMP-2 with $1 \mathrm{mM} 4$-aminophenylmercuric acetate (APMA) at $25^{\circ} \mathrm{C}$ for $2 \mathrm{~h}$ and for pro-MMP-9 at $37^{\circ} \mathrm{C}$ for $16 \mathrm{~h}$. The solutions were divided into aliquots and frozen at $-20{ }^{\circ} \mathrm{C}$ until needed.

\subsubsection{Inhibition assay}

All kinetic measurements were performed in Tris/ $\mathrm{HCl} 50 \mathrm{mM} \mathrm{pH} \mathrm{7.5,} \mathrm{CaCl}_{2} 10 \mathrm{mM}$, Brij 35 0.05\%. For each enzyme (MMP-2; cMT1; cMT3; MMP-9) the initial rate of cleavage of the fluorescent substrate Mca-Pro-LeuGly Leu-Dpa-Ala-Arg-NH2 (5 $\mu \mathrm{M}$ for MMP-2 and cMT3 and $3 \mu \mathrm{M}$ for cMT1 and MMP-9) was measured over 15-20 $\mathrm{min}$ in the absence and presence of the inhibitor at different concentrations. The percentages of inhibition (\% of inhibition) at the different inhibitor concentrations were calculated. IC50 values were obtained as previously described (O'Brien et al., 2000; Oltenfreiter et al., 2005b; Liabakk et al., 1996).

\subsection{RT PCR analysis}

The expression profile of gelatinase A and gelatinase B was determined in several human breast carcinoma cell lines (SKBR3, MDA-MB231), a mouse mammary carcinoma EF43.fgf-4 (Hajitou et al., 1998), a pancreas carcinoma (Aspc-1), a non small cellular lung carcinoma (A549), a fibrosarcoma (HT1080) and A2058, a human melanoma cells transfected with MT1-MMP cDNA (S.I.5 clone) or control vector (CIV.3 clone). Analysis was performed as described previously (Goffin et al., 2003) using RNeasy Protect Kit and QIAshredder Kit 
(QIAGEN Benelux, Venlo, Netherlands). On the basis of the results obtained, a suitable cell line to inoculate into athymic mice was chosen.

\subsection{Zymography}

\subsubsection{Tumor extracts}

Tumors were freshly frozen in $-80^{\circ} \mathrm{C}$. Proteins were extracted using $0,1 \mathrm{M}$ Tris-HCl, $\mathrm{pH} 8.1+4 \%$ Triton and using Ureum $2 \mathrm{M}$. The homogenate was centrifuged at $2000 \mathrm{~g}$ for $20 \mathrm{~min}$ at $4{ }^{\circ} \mathrm{C}$ and the supernatant stored at $-80^{\circ} \mathrm{C}$ (Remacle et al., 2000).

\subsubsection{Zymography}

Gelatinase (MMP-2 and MMP-9) activity was detected in tumor cytosols by gelatin zymography as previously described (Fabunmi et al., 1996), 15 $\mu$ g of protein were electrophoresed through a $4 \%$ polyacrylamide stacking gel and a 10\% polyacrylamide separating gel, containing $1 \mathrm{mg} / \mathrm{ml}$ gelatin (Sigma, St. Louis, MO) using a BioRad (Hercules, CA) Mini Protean II electrophoresis system. After electrophoresis, gels were incubated for $16 \mathrm{~h}$ in $50 \mathrm{mM}$ Tris- $\mathrm{HCl}(\mathrm{pH} 8.0), 150 \mathrm{mMNaCl}, 5 \mathrm{mMCaCl}_{2}, 0.05 \% \mathrm{NaN}_{3}$ at $37^{\circ} \mathrm{C}$. Gels were stained in $25 \%$ methanol, 7\% acetic acid in $\mathrm{H}_{2} \mathrm{O}$ containing 0.1\% Coomassie Brilliant Blue R250 (Biorad) and destained in the same solution in the absence of dye. Gelatinases were detected as transparent bands on the blue background of the stained gel.

\subsection{Animal experiments}

All animals were treated according to the regulations of the Belgian law and the local Ethical Committee. The guidelines of the National Institute of Health principles of laboratory animal care (NIH publication 86-23, revised 1985) were followed.

\subsubsection{Tumor model}

Three-week-old male Swiss $n u / n u$ mice were inoculated subcutaneously in the right flank (armpit region) with 5 x $10^{6}$ A549 cells. When the tumor reached a volume of approximately $1 \mathrm{~cm}^{3}$ (3-4 weeks), the mice were used for biodistribution studies and planar imaging experiments.

\subsubsection{Biodistribution studies}

Tumor bearing mice $(n=3)$ were injected intravenously in the tail vein with $740 \mathrm{kBq}(20 \mu \mathrm{Ci})$ of tracer. At 3,6 , $9,15,24$ and $48 \mathrm{~h}$ post injection mice were sacrificed by decapitation under halothane anaesthesia, their tissues excised, dried, weighed and counted for radioactivity with a Cobra automated gamma counter (Cobra II Series, Canberra Packard, Meriden, CT, USA Cobra). The injected dose (ID) was calculated by weighing the syringes before and after injection of the tracer and by the use of a dilution series of the injected tracer solution which was also weighed and counted for radioactivity. The concentration of radioactivity was decay corrected and expressed as a percentage of the injected dose per gram $(\% \mathrm{ID} / \mathrm{g})$.

\subsubsection{Metabolite analysis in blood}

NMRI mice $(n=3)$ were injected with approximately $1850 \mathrm{kBq}( \pm 50 \mu \mathrm{Ci})$ tracer i.v. in the tail vein. Mice were sacrificed $2 \mathrm{~h}$ p.i. and blood samples were collected. The samples were centrifuged for $15 \mathrm{~min}$ at $6000 \mathrm{~g}$ and the plasma was separated from the pellet. $1500 \mu \mathrm{L}$ of acetoni-trile was added per $\mathrm{g}$ of pellet, the mixture was ultrasonicated and vortexed for $15-20 \mathrm{~min}$ followed by centrifugation for $10 \mathrm{~min}$ at $6000 \mathrm{~g}$. The combined supernatant fraction was centrifuged again for $10 \mathrm{~min}$ at $6000 \mathrm{~g}$ and $750 \mu \mathrm{L}$ was processed by HPLC using an Alltech Econosil C18 column $(250 \times 10 \mathrm{~mm} ; 10 \mu \mathrm{m})$ and 60/40 acetonitrile/phosphate buffer $(0.025 \mathrm{M}$, pH 2$)$ as eluent at a flow rate of $2 \mathrm{~mL} / \mathrm{min}$ for $1^{\prime}$. For compound 2', 60/ 40 acetonitrile/phosphate buffer $(0.025 \mathrm{M}$, pH 4) at a flow rate of $2 \mathrm{~mL} / \mathrm{min}$ was used. The eluate was collected in fractions of $1 \mathrm{~mL}(=0.5 \mathrm{~min})$. These fractions were then counted for radioactivity on a Cobra automated gamma counter. To validate the extraction procedure, blood was taken from non-injected mice $(n=3)$ and spiked with $1 \mu \mathrm{Ci}$ of the labeled compound. The spiked controls underwent the same extraction procedure as mentioned above. Pellet and supernatant were counted for radioactivity in a gamma counter. The extraction yield was expressed as percentage of total radioactivity recovered in the supernatant. 


\subsubsection{Metabolite analysis in tumor tissue}

Per gram of tissue, $1500 \mu \mathrm{L}$ of acetonitrile was added. The sample was mixed for $30 \mathrm{~s}$ and centrifuged for $3 \mathrm{~min}$ at $6000 \mathrm{~g} .750 \mu \mathrm{L}$ of the supernatant was processed on a HPLC system as described above for the appropriate compounds $(n=3)$.

\subsubsection{Planar imaging}

Planar gamma camera imaging was performed in $n u / n u$ athymic mice bearing a A549 tumor using a Toshiba GCA-9300A/hg camera, equipped with a high-resolution parallel-hole collimator. All images were acquired into $256 \times 256$ matrices (FOV: $23.5 \times 12.46 \mathrm{~cm}$ ), with a photopeak window set at $15 \%$ around $159 \mathrm{keV}$ and processed with a Hermes image processing system.

Before tracer experiments were started, a $\left[{ }^{123} \mathrm{I}\right]$-HSA study was performed to measure the relative blood pool distribution in order to correct the uptake of the labeled organic compounds in the tumor area as well as the rest of the animal for bloodpool activity. Ten tumor bearing mice were injected with $7.4 \mathrm{MBq}\left[{ }^{123} \mathrm{I}\right]$-HSA and for each mouse ten dynamic planar images of $1 \mathrm{~min}$ were acquired, starting at $10 \mathrm{~min}$ after injection. Regions of interest (ROIs) were drawn around the tumor area and the contralateral background area. The tumor to contralateral background (RTB) ratios were calculated and the mean for all animals was determined.

Mice $(n=3)$ were injected intravenously with approximately $37 \mathrm{MkBq}(=1 \mathrm{mCi})$ tracer $\left(1^{\prime} ; 2^{\prime}\right)$ in the tail vein. To optimize scan time for planar scintigraphy imaging, animals were scanned at 6,24 and $48 \mathrm{~h}$ post i.v. tracer injection. During all imaging experiments, the animals were anesthetized by intraperitoneal injection (IP) of 75 $\mu \mathrm{l}$ of a solution containing $20 \mathrm{mg}$ pentobarbital per $\mathrm{ml}$ (Nembutal, $60 \mathrm{mg} / \mathrm{ml}$, Ceva Santé Animale, Belgium) and placed in the prone position in a sterile cage with filter top to preserve sterility during imaging. ROIs were drawn, RTBs were calculated and corrected for blood pool activity. By decreasing the specific activity of 1' we tried to decrease liver uptake, indicating some degree of saturable binding of the tracer in the liver and resulting in better images.

\section{Results}

The $\mathrm{IC}_{50}$ values of $(1,2)$ for MMP-2, MMP-9, cMT1 and cMT3 are expressed in $\mathrm{nM}$ and averaged $(n=3)$, standard deviations are also reported. For compound $1 \mathrm{IC}_{50}$ values were $23 \pm 3 \mathrm{M}, 429 \pm 36 \mathrm{nM} ; 180 \pm 45 \mathrm{nM}$ and $232 \pm 29 \mathrm{nM}$, respectively, and for compound 2 in $48 \pm 2 ; 740 \pm 62 ; 2509 \pm 342 ; 973 \pm 150$. The expression profiles of gelatinase A, gelatinase B in several breast carcinoma cell lines (SKBR3, MDA-MB231, EF43.fgf-4), pancreas carcinoma (Aspc-1), non small cellular lung carcinoma (A549), fibrosarcoma (HT1080) and A2058 human melanoma cells transfected with MT1-MMP cDNA (S.I.5 clone) or control vector (C.IV.3 clone) are shown in Fig. 2. Zymography on tumors grown in mice was performed and showed expression of both gelatinases (Fig. 3). Based on the results obtained, the A549 cell line was used for in vivo experiments.

Table 1: Tissue concentrations of radioactivity at various time points post i.v. administration of 2-(4'-[ $\left.{ }^{123} I\right]$ iodobiphenyl-4-sulphonylamino)-3-methyl-butyric acid (1') ${ }^{a}$ in A549 inoculated mice

\begin{tabular}{lllllll}
\hline & \multicolumn{7}{l}{ Time (h) } & \multicolumn{5}{l}{} \\
\cline { 2 - 7 } & $\mathbf{3}$ & $\mathbf{6}$ & $\mathbf{9}$ & $\mathbf{1 5}$ & $\mathbf{2 4}$ & $\mathbf{4 8}$ \\
\hline Blood & $5.93 \pm 1.24$ & $6.11 \pm 2.99$ & $6.32 \pm 1.28$ & $3.70 \pm 1.33$ & $3.31 \pm 1.54$ & $1.44 \pm 0.49$ \\
Liver & $44.86 \pm 24.25$ & $26.04 \pm 7.28$ & $28.89 \pm 5.54$ & $15.91 \pm 2.58$ & $19.62 \pm 2.99$ & $8.45 \pm 1.96$ \\
Small intestines & $3.91 \pm 0.48$ & $4.54 \pm 1.42$ & $4.48 \pm 1.2$ & $2.69 \pm 0.97$ & $2.54 \pm 0.86$ & $1.14 \pm 0.32$ \\
Large intestines & $2.43 \pm 0.27$ & $4.33 \pm 1.64$ & $5.65 \pm 2.52$ & $3.81 \pm 2.90$ & $3.11 \pm 1.20$ & $1.76 \pm 0.77$ \\
Kidneys & $8.44 \pm 2.93$ & $6.97 \pm 3.76$ & $6.92 \pm 1.55$ & $4.17 \pm 1.65$ & $4.15 \pm 1.29$ & $1.40 \pm 0.28$ \\
Stomach & $0.56 \pm 0.36$ & $0.87 \pm 0.51$ & $0.61 \pm 0.07$ & $0.56 \pm 0.36$ & $0.87 \pm 0.38$ & $0.50 \pm 0.41$ \\
Muscle & $0.79 \pm 0.65$ & $0.52 \pm 0.24$ & $0.48 \pm 0.11$ & $0.34 \pm 0.07$ & $0.32 \pm 0.18$ & $0.16 \pm 0.07$ \\
Tumor & $2.00 \pm 0.40$ & $1.83 \pm 0.42$ & $1.88 \pm 0.34$ & $1.52 \pm 0.59$ & $1.33 \pm 0.79$ & $0.75 \pm 0.44$ \\
Fat & $2.65 \pm 2.18$ & $1.76 \pm 1.06$ & $1.23 \pm 0.42$ & $0.75 \pm 0.18$ & $0.96 \pm 0.71$ & $0.37 \pm 0.08$ \\
Tumor/blood & 0.34 & 0.30 & 0.3 & 0.41 & 0.40 & 0.52 \\
Tumor/muscle & 2.52 & 3.51 & 3.92 & 4.44 & 4.13 & 4.63 \\
\hline
\end{tabular}

${ }^{a}$ Animals were injected intravenously with $740 \mathrm{kBq}\left[{ }^{123} \mathrm{I}\right] 2-\left(4^{\prime}-\left[{ }^{123} \mathrm{I}\right]\right.$ iodo-biphenyl-4-sulphonylamino)-3-methyl-butyric acid (1') and sacrificed at designated times. Units are expressed as \% injected dose/g of tissue $(\mathrm{n}=3)$ corrected for background radiation and averaged. Below are reported the standard deviations. 
Fig. 2: The expression profile of gelatinase $A$ and gelatinase $B$ in several breast carcinoma cell lines (SKBR3, MDA-MB231, EF43fgf4), pancreas carcinoma (Aspc-1), lung carcinoma (A549), fibrosarcoma (HT1080) and A2058 human melanoma cells transfected with MT1-MMP cDNA (S.I.5 clone) or control vector (C.IV.3 clone).

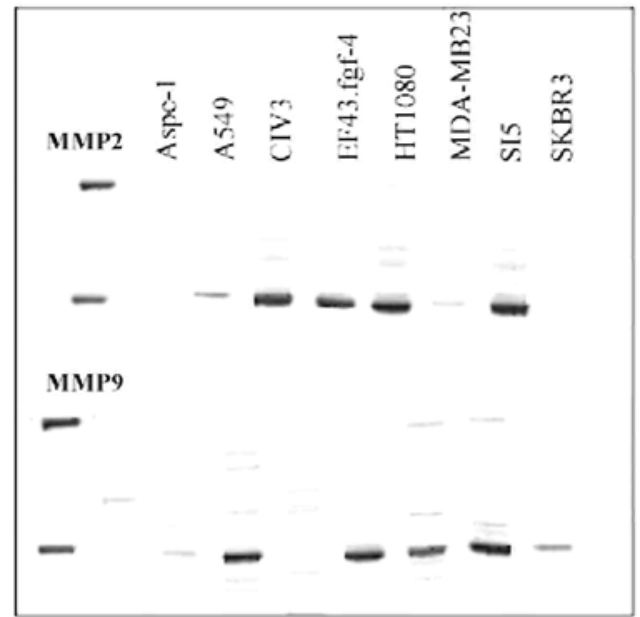

Fig. 3: Proteins were extracted using $0.1 \mathrm{M}$ Tris- $\mathrm{HCl}$, pH $8.1+4 \%$ Triton (A) and using Ureum $2 \mathrm{M}(\mathrm{B})$. The homogenate was centrifuged at $2000 \mathrm{~g}$ for $20 \mathrm{~min}$ at $4^{\circ} \mathrm{C}$ and the supernatant stored at $-80^{\circ} \mathrm{C}$. Zymography was performed as described in Section 2.
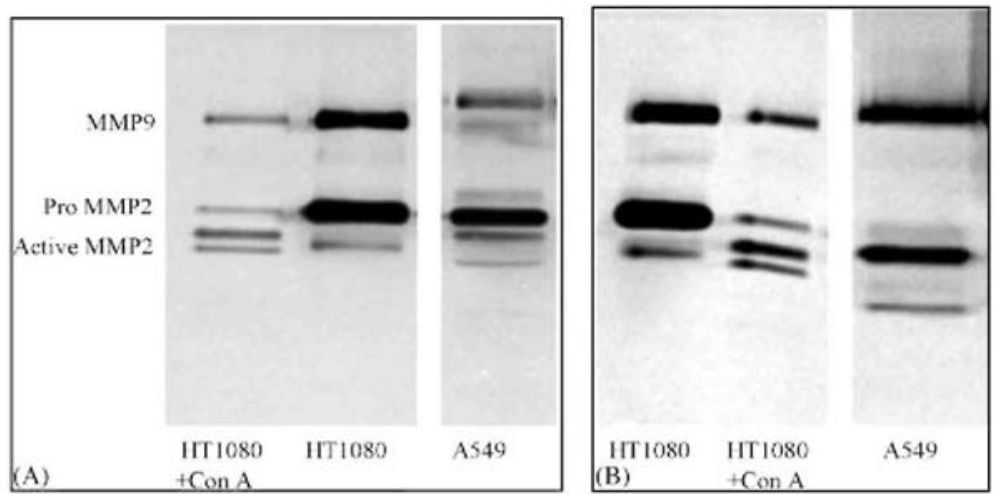

In vivo experiments showed a tumor uptake of $2.0 \pm 0.4 \% \mathrm{ID} / \mathrm{g}$ at $3 \mathrm{hp} .1$. decreasing to $0.8 \pm 0.4 \% \mathrm{ID} / \mathrm{g}$ at $48 \mathrm{hp} .1$., a tumor/blood ratio of 0.5 and a tumor/muscle ratio of 4.6 at $48 \mathrm{hp}$.i. for compound 1'. For compound $2^{\prime}$ a tumor uptake of $0.7 \pm 0.1 \% \mathrm{ID} / \mathrm{g}$ at $3 \mathrm{hp} . \mathrm{i}$. decreasing to $0.2 \pm 0.1 \% \mathrm{ID} / \mathrm{g}$ at $48 \mathrm{hp} . \mathrm{i}$, a tumor/blood ratio of 1.04 and a tumor/muscle ratio of 1.57 at $48 \mathrm{~h}$ p.i. was observed (Table $1\left(1^{\prime}\right)$ and Table $\left.2\left(2^{\prime}\right)\right)$.

HPLC analysis of the mouse plasma (extraction yields for $1^{\prime}$ and $2^{\prime} \geq 90 \%$ ) showed no dehalogenation of $1^{\prime} 2 \mathrm{~h}$ post injection. No other metabolites were detected. Therefore, the high liver uptake was not caused by metabolisation. For compound 2', 65.4\% of intact compound was found in the blood and only one polar metabolite $(31 \%)$ was detected. In the tumor $91.8 \%$ of the detected activity was represented by the intact compound and only $8.1 \%$ by a metabolite (Fig. 4 ).

The ratio between the blood flow in the tumor and the contralateral reference leg using $\left[{ }^{123} \mathrm{I}\right]$-HSA resulted in $\mathrm{RTB}=1.2 \pm 0.03$, indicating a significant higher blood pool in tumor than in reference leg. $2-\left(4^{\prime}-\left[{ }^{123} \mathrm{I}\right]\right.$ iodobiphenyl-4-sulphonylamino)-3-methyl-butyric acid (1') and 2-(4'-[ $\left.{ }^{123} \mathrm{I}\right]$ iodo-biphenyl-4-sulphonylamino $)-3-$ methyl-bu-tyramide (2') gave a slightly visible tumor uptake which was more defined when reducing specific activity of compound 1' (Fig. 4) resulting in lower liver uptake, thus indicating some degree of saturable binding of the tracer in the liver. ROIs were drawn, RTB and correction for bloodpool activity were calculated resulting 
in the following ratio's: $2.18 \pm 0.65 ; 1.65 \pm 0.48 ; 1.85 \pm 0.15$ for compound 1 ' at 6,24 and $48 \mathrm{~h}$ post injection, respectively. For compound 2 ' the corresponding ratios were $1.42 \pm 0.31 ; 1.78 \pm 0.17 ; 2.09 \pm 0.38$ at 6,24 and 48 $\mathrm{h}$ post injection. An image with reduced specific activity of 1 ' revealed no better RTB ratios: $(1.40 \pm 0.13 ; 1.62 \pm$ $0.37 ; 1.58 \pm 0.31$ ) but image quality was improved by reduced liver uptake (a decrease from $44.86 \pm 24.25 \% \mathrm{ID} / \mathrm{g}$ 3hp.i. to $12.09 \pm 1.94$ 3hp.i.) (Fig. 5).

Fig. 4: Metabolite analysis in blood, tumor of $1^{\prime}(A, C)$ and $2^{\prime}(B, D)$.
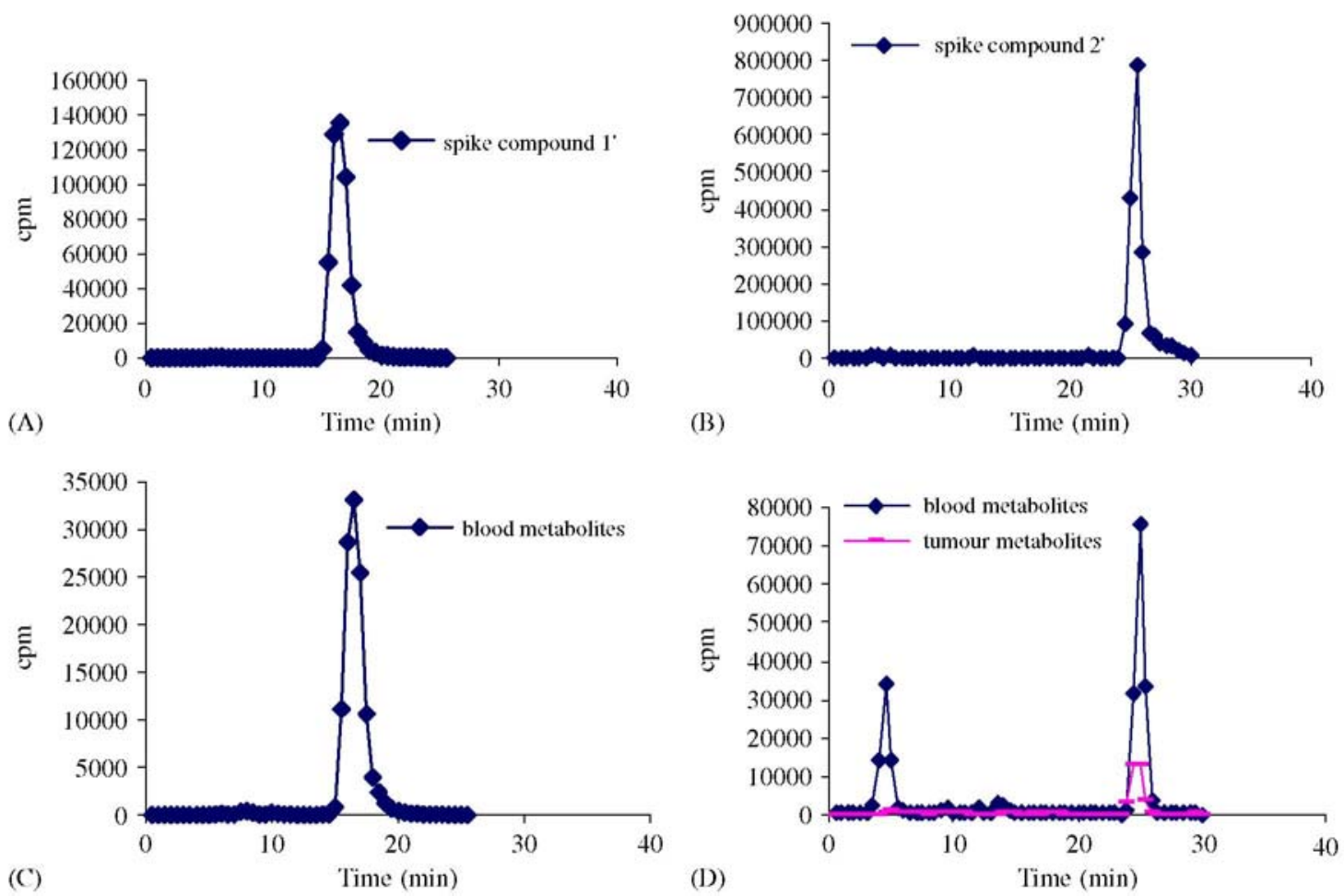

Table 2: Tissue concentrations of radioactivity at various time points post i.v. administration of 2-(4'-[ ${ }^{123}$ I] iodobiphenyl-4-sulphonylamino)-3-methyl-butyramide $\left(2^{\prime}\right)^{a}$ in A549 inoculated mice

Time (h)

\begin{tabular}{llllll}
\hline $\mathbf{3}$ & $\mathbf{6}$ & $\mathbf{9}$ & $\mathbf{1 5}$ & $\mathbf{2 4}$ & $\mathbf{4 8}$ \\
\hline $2.31 \pm 0.44$ & $1.67 \pm 0.42$ & $1.20 \pm 0.28$ & $0.49 \pm 0.17$ & $0.36 \pm 0.08$ & $0.17 \pm 0.04$ \\
$4.02 \pm 0.61$ & $3.78 \pm 0.28$ & $3.29 \pm 0.20$ & $1.98 \pm 1.06$ & $1.87 \pm 1.07$ & $0.84 \pm 0.22$ \\
$5.6 \pm 2.88$ & $3.37 \pm 1.48$ & $2.19 \pm 0.71$ & $1.4 \pm 0.65$ & $0.91 \pm 0.29$ & $0.46 \pm 0.09$ \\
$29.66 \pm 3.47$ & $11.81 \pm 4.51$ & $5.96 \pm 3.05$ & $2.84 \pm 2.39$ & $1.25 \pm 0.38$ & $0.87 \pm 0.38$ \\
$3.69 \pm 0.60$ & $3.68 \pm 0.44$ & $3.04 \pm 0.62$ & $1.46 \pm 0.68$ & $0.97 \pm 0.37$ & $0.46 \pm 0.23$ \\
$0.67 \pm 0.25$ & $1.11 \pm 0.16$ & $0.68 \pm 0.15$ & $0.51 \pm 0.29$ & $0.50 \pm 0.34$ & $0.42 \pm 0.05$ \\
$0.49 \pm 0.1$ & $0.47 \pm 0.07$ & $0.49 \pm 0.05$ & $0.36 \pm 0.21$ & $0.24 \pm 0.1$ & $0.11 \pm 0.03$ \\
$0.71 \pm 0.08$ & $0.80 \pm 0.1$ & $0.83 \pm 0.05$ & $0.53 \pm 0.23$ & $0.44 \pm 0.15$ & $0.17 \pm 0.08$ \\
$1.03 \pm 0.23$ & $0.81 \pm 0.12$ & $1.09 \pm 0.20$ & $0.51 \pm 0.16$ & $0.31 \pm 0.11$ & $0.12 \pm 0.06$ \\
0.31 & 0.48 & 0.69 & 1.07 & 1.23 & 1.04 \\
1.45 & 1.70 & 1.67 & 1.48 & 1.82 & 1.57
\end{tabular}

${ }^{\mathrm{a}}$ Animals were injected intravenously with $740\left[{ }^{123} \mathrm{I}\right] 2-\left(4^{\prime}-\left[{ }^{123} \mathrm{I}\right]\right.$ iodo-biphenyl-4-sulphonylamino)-3-methyl-butyramide $\left(2^{\prime}\right){ }^{\mathrm{a}}$ and sacrificed at designated times. Units are expressed as \% injected dose/g of tissue $(\mathrm{n}=3)$ corrected for background radiation and averaged. Below are reported the standard deviations. 
Fig. 5: (A) planar scintigraphy imaging for compounds $1^{\prime}$ and 2', respectively at 6; 24 and 48 h post i.v. tracer injection. Tumor position is pointed with an arrow. (B) Planar scintigraphy imaging for compound $1^{\prime}$ at $24 \mathrm{~h}$ post i.v. tracer injection, with reduced specific activity. Tumor position is pointed with an arrow, for scaling purposes, abdominal uptake is masked out.

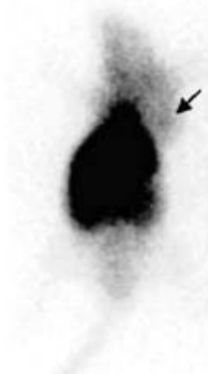

I' $(6 \mathrm{~h})$

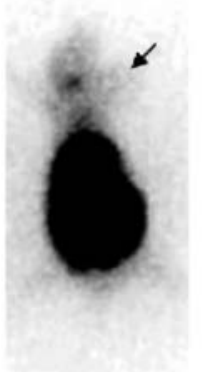

$2^{\prime}(6 \mathrm{~h})$

(A)

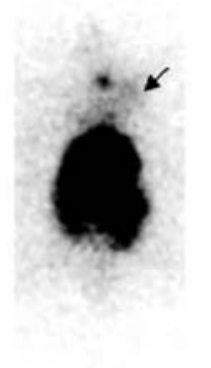

$1^{\prime}(24 \mathrm{~h})$

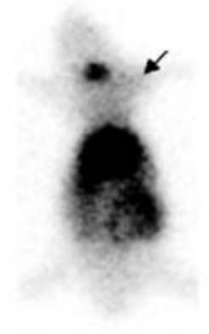

$2^{\prime}(24 \mathrm{~h})$

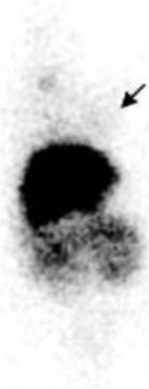

I' (48 h)

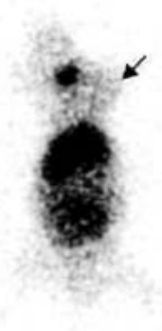

$2^{\prime}(48 \mathrm{~h})$

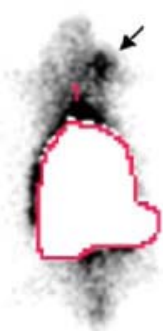

1' (24h)

(B)

\section{Discussion}

The MMPIs all share a common scaffold: a peptidomimetic backbone that interacts with "substitutes" surrounding the active site coupled to a metal chelator (zinc-binding group) that binds to the catalytic $\mathrm{Zn}$ (II) ion. To improve the selectivity of MMPIs, this backbone is substituted with side chains that interact with specific pockets within the active domain of the MMPs (Fei et al., 2003). The S1' subsite for the gelatinases is relatively deeper than for other subtypes and is targeted to obtain highly selective inhibitors. A sulphonamide group is incorporated in the inhibitor to improve the enzyme-inhibitor binding not only by forming hydrogen bonds to the enzyme but also by properly directing the hydrophobic substituent to the S1' subsite and enabling it to plunge in deeply (Kiyama et al., 1998). The biphenyl function is incorporated to improve selectivity towards gelatinases. Tryptophane-based biphenylsulphonamide matrix metalloproteinase inhibitors were synthesized, evaluated in vitro; in vivo and as imaging agents and published previously [14]. Structural changes to the biphenyl ring system, in the aposition to carboxylic/hydroxamic acid, and to the carboxylate moiety have an influence on biological activity, selectivity and pharmacokinetic properties. Accordingly, we developed valine-based biphenylsulphonamide matrix metalloproteinase inhibitors, which were evaluated in the current paper. The $\mathrm{IC}_{50}$ 
values of all synthesized analogues were in the nanomolar range and thus suitable for in vivo imaging. There was, however, a distinction between the valine and the earlier published tryptophane derivatives, namely that both hydroxamic and carboxylic acid Valine compounds showed a higher selectivity for MMP-2 in comparison to the Tryptophane analogues, which is probably reflected in a different inhibition of tumor invasiveness due to the importance of especially MMP-2. Metabolite analysis revealed a lower dehalogenation of valine analogues, probably resulting in higher tumor uptake and slightly better images, whereas with tryptophane only a high abdominal uptake could be detected while a lower tumor uptake was detected. As for both compounds no or less metabolites are present in tumor tissue and uptake in the tumor could not be attributed to blood pool activity, the blinding of the evaluated MMPIs may be specific and there could be a future for other small organic MMP inhibitors. Acceptable tumor uptake of valine compounds combined with the relatively rapid wash-out from normal tissues, excluding the liver, should allow for straightforward imaging of tumors and thus support the theoretical suitability of these tracers in vivo. The results have shown however that other parameters must be looked at, and that the discrepancy between encouraging in vitro results and in vivo results (rather low tumour uptake and doubtful use as imaging agent) was also evidenced by others and remains to be elucidated (Zheng et al., 2004; Zheng et al., 2003; Furumoto et al., 2003).

So far, it it has been difficult to use MMPIs as tumor imaging agents, however, new analogues in combination with other tumour models are mandatory to better understand and evaluate MMPIs as imaging agents. ${ }^{18} \mathrm{~F}$ labeled analogues could be produced to obtain higher resolution of images. The possibility of visualizing tumors will also depend largely upon their MMP expression profile which varies over time. Thus, prior to clinical evaluation, additional research focusing on the influence of tumor stage, in various types of tumor models with known overexpression of MMPs, on uptake of newly synthesised analogues; as well as the relationship between tracer uptake and MMP expression is mandatory (Furumoto et al., 2003).

\section{Acknowledgment}

The authors gratefully acknowledge support from The European Union Framework Programme 6 (LSHC-CT2003-503297 'Cancerdegradome') and wish to thank D. Delapierre of the Laboratory of Tumor and Developmental Biology, University of Liège, Sart-Tilman, for her great help with the determination of the in vitro MMP inhibitory activity of all synthesised compounds.

\section{References}

Bernhard, E.J., Gruber, S.B., Muschel, R.J., 1994. Direct evidence linking expression of matrix metalloproteinase 9 (92 kDa gelatinase/collage-nase) to the metastatic phenotype in transformed rat cells. Proc. Natl. Acad. Sci. 91, 4293-4297.

Bremer, C, Tung, C.H., Weissleder, R., 2001. In vivo molecular target assessement of matrix metalloproteinase inhibition. Nat. Med. 7, 743748 .

Davies, B., Waxman, J., Wasan, P., 1993. Levels of matrix metallopro-teinases in bladder cancer correlate with tumor grade and invasion. Cancer Res. 53, 5365-5369.

Edwards, D.R., Murphy, G., 1998. Cancer: proteases-invasion and more. Nature 394, 527-528.

Edwards, D.R., Beaudry, P.P., Laing, T.D., Kowal, V., Leco, K.J., Lim, M.S., 1996. The roles of tissue inhibitors of metalloproteinases in tissue remodelling and cell growth. Int. J. Obesity 3 (suppl), S9.

Fabunmi, R.P., Baker, A.H., Murray, E.J., Booth, R.F.G., Newby, A.C., 1996. Divergent regulation by growth factors and cytokines of 95 $\mathrm{kDa}$ and $72 \mathrm{kDa}$ gelatinases and tissue inhibitors of metalloproteinases-1, -2 and -3 in rabbit aortic smooth muscle cells. Biochem. J. 315 , $335-342$.

Fang, J., Shing, Y., Wiederschain, D., et al., 2000. Matrix metalloprotei-nase-2 is required for the switch to the angiogenic phenotype in a tumor model. Proc. Natl. Acad. Sci. 97, 3884-3889.

Fei, X., Zheng, Q.H., Liu, X., Wang, J.Q., Sun, H.B., Mock, B.H., Stone, K.L., Miller, K.D., Sledge, G.W., Hutchins, G.D., 2003. Synthesis of radiolabeled biphenylsulphonamide matrix metalloproteinase inhibitors as new potential PET cancer imaging agents. Bioorg. Med. Chem. Lett. 13, 2217-2222.

Folkman, J., 1997. Angiogenic zip code. Nat. Biotechnol. 17, 749. 
Goffin, F., Munaut, C, Frankenne, F., d'Hauterive, S.P., Béliard, A., Fridman, V., Nervo, P., Colige, A., Foidart, J.M., 2003. Expression pattern of metalloproteinases and tissue inhibitors of matrix-metallo-proteinases in cycling human endometrium. Biol. Reprod. 69 (3), 976984.

Hajitou, A., Baramova, E.N., Bajou, K., Noe, V., Bruyneel, E., Mareel, M., Collette, L, Foidart, J.M., 1998. Calberg-Bacq CM. FGF-3 and FGF-4 elicit distinct oncogenic properties in mouse mammary myoepithelial cells. Oncogene 17 (16), 2059-2071.

Hanessian, S., Moitessier, N, Gauchet, C, Viau, M., 2001. N-Aryl sulphonyl homocysteine hydroxamate inhibitors of matrix metalloproteinases : further probing of the $\mathrm{S}_{1}$ : $\mathrm{S}_{1}{ }^{\prime}, \mathrm{S}_{2}{ }^{\prime}$ pockets. J. Med. Chem. 44, 3066-3073.

Heppner, K.L, Matrisian, L.M., Jensen, R.A., Rodgers, W.H., 1996. Expression of most MMP family members in breast cancer represents a tumor-induced host response. Am. J. Pathol. 149, 273-282.

Kiyama, R., Tamura, Y., Watanabe, F., Tsuzuki, H., Ohtani, M., Yodo, M., 1998. Homology modeling of gelatinase catalytic domains and docking simulations of novel sulfonamide inhibitors. J. Med. Chem. 42, 1723-1738.

Kontogiorgis, C.A., Papaioannou, P., Hadjipavlou-Litina, D.J., 2005. Matrix metalloproteinase inhibitors: a review on pharmacophore mapping and (Q)SARs results. Curr. Med. Chem. 12 (3), 339-355.

Kossakowska, A.E., Urbanski, S.J., Huchcroft, S.A., Edwards, D.R., 1992. Relationship between the clinical aggressiveness of large cell immunoblastic lymphomas and expression of $92 \mathrm{kDa}$ gelatinase (type IV collagenase) and tissue inhibitor of metalloproteinases-1 (TIMP-1) RNAs. Oncol. Res. 4, 204-233.

Lang, R., Braun, M., Sounni, N.E., Noel, A., Frankenne, F., Foidart, J.M., Bode, W., Maskos, K., 2004. Crystal structure of the catalytic domain of MMP-16/MT3-MMP: characterization of MT-MMP specific features. J. Mol. Biol. 336 (1), $213-225$.

Liabakk, N.B., Talbot, I., Smith, R.A., Wilkinson, K., Balkwill, K., 1996. Matrix metalloproteinase 2 (MMP-2) and matrix metalloproteinase 9 (MMP-9) type IV collagenaes in colorectal cancer. Cancer Res. 56, 190-196.

MacDougall, J.R., Matrisian, L.M., 1995. Contributions of tumor and stromal matrix metalloproteinases to tumor progression, invasion and metastasis. Cancer Metastasis Rev. 14, 351-362.

Murray, G.I., Duncan, M.E., O'Neil, P., Melvin, W.T., Fothergill, J.E., 1996. Matrix metalloproteinase-1 is associated with poor prognosis in colorectal cancer. Nat. Med. 2, 461-462.

O'Brien, P.M., Ortwine, D.F., Pavlovsky, A.G, Picard, J.A., Sliskovic, D.R., Roth, B.D., Dyer, L.L., Johnson, R.D., Man, CF., Hallak, H., 2000. Structure-activity relationships and pharmacokinetic analysis for a series of potent, systemically available biphenylsulphonamide matrix metalloproteinase inhibitors. J. Med. Chem. 43, 156-166.

Oltenfreiter, R., Staelens, L., Lejeune, A., Dumont, F., Frankenne, F., Foidart, J.M., Siegers, G, 2004. New radioiodinated carboxylic and hydroxamic MMP inhibitor tracers as potential tumor imaging agents. Nucl. Med. Biol. 31 (4), 459-468.

Oltenfreiter, R., Staelens, L., Labied, S., Kersemans, V., Frankenne, F., Noël, A., Van de Wiele, C, Siegers, G, 2005a. Tryptophane-based biphenylsulfonamide matrix metalloproteinase inhibitors as tumor imaging agents. Cancer Biother. Radiopharm. 6 (20), 639-647.

Oltenfreiter, R., Staelens, L., Lejeune, A., Heremans, A., Frankenne, F., Foidart, J.M., Siegers, G, 2005b. Radioiodinated biphenyl carboxylic and hydroxamic MMP inhibitor tracers as potential tumor imaging agents. Appl. Radiat. Isotopes 62 (6), 903.

Remacle, A., McCarthy, K., Noël, A., Maguire, T., McDermott, E., O'Higgins, N, Foidart, J.M., Duffy, M.J., 2000. High levels of TIMP-2 correlate with adverse prognosis in breast cancer. Int. J. Cancer 89 (2), 118-121.

Rudek, M.A., Venitz, J., Figg, W.D., 2002. Matrix metalloproteinase inhibitors: do they have a place in anticancer therapy? Pharmacotherapy 22 (6), 705-720.

Skiles, J.W., Gonnella, N.C., Jeng, AY., 2001. The design, structure, and therapeutic application of matrix metalloproteinase inhibitors. Curr. Med. Chem. 8, 425-474.

Tamura, Y., Watanabe, F., Nakatani, T., Yasui, K., Fuji, M., Komurasaki, T., Tsuzuki, H, Maekawa, R., Yoshioka, T., Kawada, K., Sugita, K., Ohtani, M., 1998. Higly selective and orally active inhibitors of type IV collagenase (MMP-9 and MMP-2): N-sulphony-lamino acid derivatives. J. Med. Chem. 41, 640-649.

Zheng, Q.H., Fei, X., DeGrado, T.R., Wang, J.Q., Stone, K.L., Martinez, T.D., Gay, D.J., Baity, W.L., Mock, B.H., Glick-Wilson, B.E., Sullivan, M.L., Miller, K.D., Sledge, G.W., Hutchins, G.D., 2003. Synthesis, biodistribution and micro-PET imaging of a potential cancer biomarker carbon-11 labeled MMP inhibitor (2R)-2-[[4-(6-nuorohex-1-ynyl)phenyl]sulfonylamino]-3-methylbutyric acid [11C] methyl ester. Nucl. Med. Biol. 30 (7), 753-760.

Zheng, Q.H., Fei, X., Liu, X., Wang, J.Q., Stone, K.L., Martinez, T.D., Gay, D.J., Baity, W.L., Miller, K.D., Sledge, G.W., Hutchins, G.D., 2004. Comparative studies of potential cancer biomarkers carbon-11 labeled MMP inhibitors (S)-2-(4'-[11C]methoxybiphenyl-4sulfonylamino)-3-methylbutyric acid and N-hydroxy-(R)-2-[[(4'-[11C]methoxyphenyl)sulfo-nyl]benzylamino]-3-methylbutanamide. Nucl. Med. Biol. 31 (1), 77-84 